\title{
Fatal Upper Gastrointestinal Bleed Following Endoscopic Biopsy of Testicular Germ Cell Tumor Metastasis to Duodenum
}

\author{
Gull Mohd. Bhat, Shiekh Aejaz Aziz*, A.R.Lone, Manzoor Ahmad Banday and Syed Nisar \\ Department of Medical Oncology, Sher-i-Kashmir Institute of Medical Sciences, Srinagar and Department of \\ Medical Oncology, IRCH, All India Institute of Medical Sciences, New Delhi.
}

\section{Introduction}

Testicular germ cell tumor, choriocarcinoma and malignant melanoma are highly vascular tumors that tend to bleed when metastatic to gastrointestinal tract. Diagnosis of germ cell tumor metastatic to gastrointestinal tract should be considered when a known patient of germ cell tumor of testis (or ovary) presents with a gastrointestinal bleed. GI bleed can occur spontaneously or following endoscopic biopsy of the lesion. This could be a rare cause of gastrointestinal bleed in a young patient and, exceptionally, this may be the first presentation of this disease. A young adult with of GCT of testis developing recurrence in the form of duodenal metastasis is discussed. Till now only a few similar cases are reported in literature.

\section{Case Report}

DMS, a 30 year male started with right testicular swelling in December 2004. He was operated in a rural district hospital and referred to the outpatient department with symptoms of bilateral flank pains, fever and sweating and passage of black tarry stools. On examination, he was pale, had a left supraclavicular node which was hard and fixed. Per abdominal examination revealed a hard mass in umbilical and epigastric region, which roughly measured $12 \times 10 \mathrm{~cm}$. Rest of the general physical and systemic examiantion was normal. Investigative profile showed a

Reprint Request:

Dr. Gull Mohd. Bhat

Associate Professor

Department of Medical Oncology

SKIMS, P.B. 27, Srinagar-190011 J\&K India.
$\mathrm{Hb}$ of $6 \mathrm{~g} / \mathrm{dl}$; TLC of $5.43 \times 10^{9} / 1$, DLC-N-85, L 11.2, M-4, MCV-81.7 fl, Platelet $281 \times 10^{9} / 1$. Serum levels of urea, creatinine, bilirubin, AST, ALT were normal. Proteins were $5.7 \mathrm{~g} / \mathrm{dl}$ (normal5.5-7.0g/dl) and albumin $2.5 \mathrm{~g} / \mathrm{dl}$ (normal $3.5-5.6 \mathrm{~g} / \mathrm{dl}$ ). Uric acid was $3.9 \mathrm{mg} / \mathrm{dl}$ (normal 3.4 - $6.5 \mathrm{mg} / \mathrm{dl}$ ) and LDH was $3310 \mathrm{u} / 1$ (normal 240-480 u/l). Alpha feto protein level was-3391.63u/1 and HCG levels were persistently more than $100 \mathrm{iu} / 1$. Chest X-Ray was normal. USG scan of the abdomen showed a $6.3 \times 5.3$ mass and nodes in the paraaortic region. CECT of abdomen showed a large, heterogeneous, retroperi-toneal mass having varying contrast enhancement with vascular invasion and mild left sided hydronephrosis. FNAC of the mass was suggestive of anaplastic large cell lymphoma or germ cell tumor and that of the left cervical node showed undifferentiated tumor with marked necrosis. Gastrodudenoscopy showed ulceroproliferative lesion involving 50 percent of circumference. Review of the duodenal biopsy which was initially reported as adenocarcinoma showed features consistent with germ cell tumor. By definition patient had high risk GCT of testis. He was treated with 4 cycles of BEP chemo-therapy. All markers serially normalized. and patient was advised surgical consultation but did not follow up. Later he presented with abdominal pain. USG showed evidence of duodenal or epigastric mass. Chest X-ray showed diffuse bilateral nodular shadows. Repeat upper gastroduodeno2scopy showed ulceroinfiltrative tumor in third part of duodenum. Histopathology of the lesion showed evidence of germ cell tumor. However, patient developed hematochezia after the procedure. He did not respond to conservative measures and succumbed to this complication. 


\section{Discussion}

In young males with a known or unknown testicular GCT, presenting with GI bleed and duodenal tumor, metastasis from the primary malignancy should be strongly considered. Treatment of this condition could require a multimodality approach, not infrequently including emergency surgery. Though these patients often belong to a poor-prognosis group, the literature review shows that long-term survival is possible using modern treatment principles. The risk of GI hemorrhage, at presentation with or without treatment is particularly high. In a retrospective review of 5 cases with advanced germ cell malignancy (testicular 2 and retroperitoneal 3 ) and involvement of the upper GI tract with germ cell malignancy was proved, in 4 patients the duodenum and in 1 patient the distal part of the esophagus was involved. Three patients had grade 3 or grade 4 anemia. Ulceration of the upper GI tract was demonstrated in 1 case complicated by an aortoduodenal fistula and aortic rupture. This patient and 2 other cases needed emergency surgery due to GI hemorrhage before and /or during the initial phase of chemotherapy.' Three more cases of metastatic testicular cancer to duodenum causing intestinal bleed have been described by other authors. ${ }^{2}$ In similar reports, young patients presenting for the first time with such bleed have been reported. ${ }^{a s s}$ Hemorrhage due to such metastasis could be massive and even fatal." A case of metastatic choriocarcinoma with chronic blood loss has also been described.' Propenicity to bleed indicates high vascularity analogous to intracranial bleeds in CNS metastasis from melanoma, thyroid, renal cell carcinoma and choriocarcinoma. Other tumors involving small gut that lead to gastrointestinal bleed include, adenocarcinoma, carcinoid tumors, lymphoma, lymphangioma, gastrointestinal stromal cell tumor, lipoma and others.' The patient under discussion was managed conser-vatively but had recurrence of duodenal bleed after stabilization and died of the same. Thus it is concluded that the tumor bleed in a metastatic lesion in GCT, spontaneous or following biopsy in a young patient should not be taken lightly and active surgical resection perhaps remains the best option of treatment.

\section{References}

1. Nord, S.D. Fossa, K.E. Giercksky. Gastrointestinal Presentation of Germ Cell Malignancy in European. Urology 2000;38:721-4.

2. Veen HF, Bruining HA. Gastro-intestinal bleeding and malignancy of the testis. Acta Chir Scand 1979; 145(5):363-5.

3. Nakamura A, Ikeda Y, Morishita S, Sato Y, Matsumoto M, Inomoto T, Kariya T, Inoue T. Upper gastrointestinal bleeding arising from metastatic testicular tumor. $\mathcal{F}$ Gastroenterol 1997;32(5):650-3.

4. Malhotra P, Sriram PV, Kochhar R, Rajwanshi A, Radotra BD, Varma S. Teratocarcinoma presenting with duodenal metastases diagnosed on endoscopic fine needle aspiration. Indian F Gastroenterol 1999; 18(4):175.

5. Weidmann B, Eisenbach T, Borger R, Niederle Upper gastrointestinal bleeding as the first manifestation of a nonseminomatous testicular carcinoma. Dtsch Med Wochenschr 1996;121(46):1428-32.

6. Cunningham LN, Ginsberg P, Manfrey S, Finkelstein LH. Massive hemorrhage secondary to metastatic testicular carcinoma. J Am Osteopath 1989; 89(3):341-4.

7. Syrigos KN, Tsioulos D, Efstathiou S, Kouvaras S, Mitromaras A, Merikas EA. Metastatic testicular cancer with massive gastrointestinal haemorrhage as initial presentation. Clin Oncol (RCollRadiol) 2002;14(2):179-81.

8. Stokes EW, Perkins C Testicular choriocarcinoma. An unusual presentation as occult gastrointestinal blood loss. $\mathcal{F}$ Adolesc Health Care. 1989;10(2):146-50

9. Grubbs EG, Tyler DS. Neoplasms of the Small Intestine in Cancer Medicine, 6 the Edition, 2003;1623-1630. 